\title{
A Comparison of the Performance of Out-of-hospital Cardiac Arrest Score and Standard Severity Scores in Predicting Hospital Mortality and Neurological Consequence in Out-of-hospital Cardiac Arrest Patients
}

\author{
Natthaka Sathaporn, M.D., Bodin Khwannimit, M.D.
}

Department of Internal Medicine, Faculty of Medicine, Prince of Songkla University, Hat Yai, Songkhla 90110, Thailand.

Received 2 January 2021 • Revised 8 April 2021 • Accepted 15 April 2021 • Published online 24 June 2021

\begin{abstract}
:
Objective: There is limited data to determine the performance of general and specific severity score in out-of-hospital cardiac arrest (OHCA) patients. Hence, we compared the performance of the OHCA score with Acute Physiology and Chronic Health Evaluation (APACHE) and Simplified Acute Physiology Score (SAPS) to predict outcome in OHCA patients.

Material and Methods: A retrospective study was conducted in a mixed intensive care unit of a tertiary hospital. The primary outcome was in-hospital mortality. The secondary outcome was poor neurological outcome.

Results: A total of 190 OHCA patients were enrolled. The OHCA score had moderate discrimination with an area under the receiver operating characteristic curve (AUC) $0.77(95 \% \mathrm{Cl} 0.7-0.837)$ whereas discrimination of APACHE II-IV, SAPS II, and SAPS 3 were good with an AUC more than 0.8. The actual hospital mortality rate was $64.7 \%$. The OHCA score predicted hospital mortality of $95.3 \pm 8.4$, which significantly overestimated the mortality with standardized mortality ratio 0.68 (95\% Cl 0.56-0.81). However, all severity scores revealed poor calibration. Additionally, overall performance of APACHE II-IV, SAPS II and SAPS 3 were better than the OHCA score. For secondary outcome, discrimination of the OHCA score was moderate with an AUC $0.790(95 \% \mathrm{Cl} 0.700-0.878)$ whereas other severity scores demonstrated good discrimination with AUC more than 0.8.

Conclusion: APACHE II-IV, SAPS II, and SAPS 3 indicated superior overall performance and demonstrated good discrimination for predicting hospital mortality and unfavorable neurological consequence better than the OHCA score. However, all severity scores attested poor calibration, therefore, specific scores for OHCA patients should be modified.
\end{abstract}

Keywords: APACHE, SAPS, outcome, risk prediction

Contact: Natthaka Sathaporn, M.D.

Department of Internal Medicine, Faculty of Medicine, Prince of Songkla University, Hat Yai, Songkhla 90110, Thailand.

E-mail: bellchestcritcare@gmail.com

(c) 2021 JHSMR. Hosting by Prince of Songkla University. All rights reserved.

This is an open access article under the CC BY-NC-ND license

(http://www.jhsmr.org/index.php/jhsmr/about/editorialPolicies\#openAccessPolicy).
J Health Sci Med Res 2022;40(2):137-146 doi: $10.31584 / j h s m r .2021821$ www.jhsmr.org 


\section{Introduction}

Out-of-hospital cardiac arrest (OHCA) is a major global medical problem. The overall prognosis of OHCA varies among studies, with a low median survival rate of $2.0-20.0 \% .^{1-4}$ The survival improvement is considerable as well as the neurological outcome with less than $10.0 \%$ able to return their previous status. ${ }^{5-7}$ The prediction of neurological consequence in the survivor after cardiac arrest is extremely crucial for counselling patients' families, reducing unnecessary costs and facilitating organ donation. The discipline of intensive care medicine is particularly concerned with mortality prediction. Severity scoring system is a crucial tool for patients admitted in the intensive care unit (ICU) in order to predict hospital mortality, characterize disease severity, and compare ICU quality between time and unit performance. ${ }^{8-13}$ The non-disease specific severity scores including the Acute Physiology and Chronic Health Evaluation (APACHE) and Simplified Acute Physiology Score (SAPS) are two models currently in common use for accessing severity of ICU patients. ${ }^{9,13-16}$ Those were designed for general patients, not specific for post cardiac arrest populations. Therefore, the OHCA score was derived. The OHCA score needs only five parameters comprising estimated no-flow and low-flow intervals, initial rhythm, serum creatinine level and blood lactate, whereas APACHE and SAPS need many variables for calculation. A previous study found that the OHCA score demonstrated good discrimination and calibration for hospital mortality prediction. ${ }^{17}$

Although severity could be evaluated by either general or specific scoring system, the applications of these measurements (APACHE, SAPS, the OHCA score) depend on the clinical conditions of patients and different settings of management, and few studies have determined their performance for prognosticating in-hospital mortality and neurological consequence in patients with OHCA. Therefore, the object of this study was to verify the performance between the OHCA score versus APACHE II-IV ${ }^{18-21}$, SAPS $\mathrm{I}^{22}$, and SAPS $3^{23}$, in patients with OHCA and return of spontaneous circulation (ROSC).

\section{Material and Methods}

A retrospective analysis of data all adult OHCA patients admitted in the mixed ICUs of a referral medical center in Southern Thailand was conducted. The study was approved by our institutional ethics committee (REC. 62-301-14-1).

Consecutive critically ill adult patients (>18 years) were diagnosed OHCA with ROSC admitted to our ICUs from January 2011 to September 2019 and enrolled. Cardiac arrest is defined as the absence of palpable pulse with the absence of spontaneous breathing. ROSC was defined as recovery of blood pressure and pulse for more than one hour, with or without administration of vasopressor or inotropic agents. ${ }^{17}$ Patients who did not have serum lactate level within twenty-four hours after admission were excluded because it is one of the parameters for calculating the OHCA score. Besides, patients whose cause of cardiac arrest was due to trauma were excluded.

The outcomes were survival status on hospital discharge and Cerebral Performance Category (CPC) of the Glasgow-Pittsburgh Outcome Categories. All parameters required by APACHE, SAPS and OHCA scores as determined in the original articles were collected for analysis. The physiological variables in APACHE $\|-I^{18-21}$ and SAPS $\|^{22}$ were calculated based on the worst values within the first 24 hour after ICU admission, in contrast to the physiological data of SAPS $3^{23}$, which were based on the worst values before or after the first hour of ICU admission. The biochemical variables for OHCA score were obtained at ICU admission in patients who return to spontaneous circulation, before the further interventions were performed. The primary outcome was hospital mortality. Poor neurological consequence assessed by 
using CPC was the secondary outcome. Poor neurological outcome was identified by CPC score of 3 to 5 . $^{6,24,25}$

To estimate the sample size, we assumed the performance of the OHCA score may not differ more than $15.0 \%$ when compared to other non-disease specific scores such as APACHE and SAPS. Based on the original study, the area under the receiver operating characteristic curve (AUC) of the OHCA score was $0.88 .^{17}$ This study needed 135 patients to achieve $80.0 \%$ power and $5.0 \%$ type I error.

Continuous variables were presented as means with standard deviations or median with interquartile range, and categorical variables were reported as numbers and percentages. Chi-square and Wilcoxon rank sum test were used to compare category variables and continuous variables, respectively. The performance of the severity scores was appraised by discrimination, calibration, and overall performance. The discrimination refers to the ability of the score to discriminate between survivors and nonsurvivors. This index was evaluated by using the AUC. ${ }^{15}$ An AUC greater than 0.8 indicates good discrimination, while 0.7-0.8 indicates moderate discrimination, and 0.5-0.7 indicates low discrimination. ${ }^{26}$ Calibration evaluates the rank of agreement between expected probabilities of mortality and actual mortality across all of the strata of probabilities of death. Calibration was examined by the Hosmer-Lemeshow $(\mathrm{H}-\mathrm{L})$ goodness-of-fit $\mathrm{H}$ and $\mathrm{C}$ statistics, and standardized mortality ratio (SMR). ${ }^{10,15}$ Patients were rank-ordered in five groups according to their probability of death to calculate the $\mathrm{H}$ statistic. To evaluate the $\mathrm{C}$-statistic, the patients were divided into quintiles of predicted risk, $p$-value $>0.100$ indicated goodness-of-fit. ${ }^{17}$ The SMR is a ratio that is calculated by dividing the observed number of non-survivors in the study group and the number of non-survivors which would be anticipated from severity score. Therefore, $S M R>1$ is an underestimation, whereas $\mathrm{SMR}<1$ is an overestimation of the mortality by the severity scoring system prediction. The Brier score refers overall performance including both discrimination and calibration. ${ }^{10}$ The lower Brier score is shown, the higher accuracy of severity score is represented. All statistical analysis was performed using Stata version 11.

\section{Results}

There were $271 \mathrm{OHCA}$ with ROSC patients admitted in ICU during the study period. After applying the exclusion criteria, 81 patients were excluded because of lack of serum lactate level $(n=47)$ and traumatic patients $(n=34)$. Overall, 190 patients were included for analysis.

There was some difference in the baseline characteristics of the patients between survivors and non-survivors in terms of age, assumed arrest due to cardiac cause, revascularization in myocardial infarction, duration of no flow time, shockable rhythm and serum lactate (Table 1).

A total of 190 patients were analyzed, survivors were 67 cases and non-survivors were 123 cases. The quantity of male gender was higher than female gender in both groups. The majority situation of cardiac arrest occurred at home (65.8\%). Myocardial infarction was the most common cause of arrest in survivors (40.3\%), whereas it was the second most common cause in non-survivors (27.6\%), and hypoxic arrest was the main cause in non-survivors (30.0\%). The proportion of myocardial infarction patients which received revascularization was 19/27 (70.4\%) in survivors and 9/34 $(26.5 \%)$ in non-survivors. Therapeutic hypothermia was provided in 20 cases of survivors $(29.9 \%)$ and 28 cases $(22.8 \%)$ in non-survivors. The percentage of VA ECMO was equipped 3 and 3.3 in survivors and non-survivors, correspondingly.

Hospital mortality rate as sorted by the OHCA score are shown in Figure 1. The mortality rate substantially increased in patients with higher scores. The OHCA score of 31-50 had percentage of mortality rate at fifty-four. Furthermore, the OHCA score of 51-60 had a mortality rate of $76.5 \%$, rising to $91.0 \%$ in cases of $\mathrm{OHCA}$ score more 
than 60. Discrimination of the OHCA score was moderate with the AUC $0.77(95 \% \mathrm{Cl} 0.7-0.837)$. On the contrary, the non-disease specific severity scores such as APACHE
II-IV, SAPS II and SAPS 3 had good discrimination with the AUC more than 0.8 (Figure 2).

Table 1 Clinical demographic data

\begin{tabular}{|c|c|c|c|c|}
\hline Parameters & $\begin{array}{l}\text { All patients } \\
(n=190)\end{array}$ & $\begin{array}{l}\text { Survivors } \\
(n=67)\end{array}$ & $\begin{array}{l}\text { Non-survivors } \\
(n=123)\end{array}$ & $p$-value \\
\hline Age, years (IQR) & $60(47.0-77.0)$ & $58(43.0-68.0)$ & $62(52.0-75.0)$ & 0.030 \\
\hline Male $[\mathrm{n}(\%)]$ & $122(64.2)$ & $42(62.7)$ & $80(65.0)$ & 0.750 \\
\hline Witness arrest [n (\%)] & $170(89.5)$ & $64(95.5)$ & $106(86.2)$ & 0.050 \\
\hline \multicolumn{5}{|l|}{ Place of event [n (\%)] } \\
\hline Home & $125(65.8)$ & $37(55.2)$ & $88(71.5)$ & 0.023 \\
\hline Public & $51(26.8)$ & $24(35.8)$ & $27(22.0)$ & 0.039 \\
\hline During transfer with personnel & $14(7.4)$ & $6(9.0)$ & $8(6.5)$ & 0.537 \\
\hline Bystander [n (\%)] & $52(27.4)$ & $21(31.3)$ & $31(25.2)$ & 0.360 \\
\hline Assume cardiac cause [n (\%)] & $85(44.7)$ & $40(59.7)$ & $45(36.6)$ & 0.002 \\
\hline \multicolumn{5}{|l|}{ Cause $[\mathrm{n}(\%)]$} \\
\hline Myocardial Infarction & $61(32.1)$ & $27(40.3)$ & $34(27.6)$ & 0.074 \\
\hline Hypoxic & $52(27.4)$ & $15(22.4)$ & $37(30.0)$ & 0.256 \\
\hline Arrhythmia & $17(8.9)$ & $10(14.9)$ & $7(5.7)$ & 0.033 \\
\hline Sepsis & $16(8.4)$ & $1(1.5)$ & $15(12.2)$ & 0.011 \\
\hline Other* & $38(20.0)$ & $14(20.9)$ & $24(19.5)$ & 0.820 \\
\hline Unknown & $6(3.2)$ & $0(0.0)$ & $6(5.0)$ & 0.066 \\
\hline Adrenaline dose before ROSC, mg (IQR) & $3(2.0-7.0)$ & $3(2.0-9.0)$ & $3(3.0-7.0)$ & 0.210 \\
\hline Therapeutic temperature management [n (\%)] & $48(25.3)$ & $20(29.9)$ & $28(22.8)$ & 0.280 \\
\hline Myocardial infarction with revascularization [n (\%)] & $28(45.9)$ & $19(70.4)$ & $9(26.5)$ & $<0.001$ \\
\hline VA ECMO [n (\%)] & $6(3.2)$ & $2(3.0)$ & 4 (3.3) & 0.920 \\
\hline ICU LOS, days (IQR) & $3(2.0-7.0)$ & $6(3.0-10.0)$ & $3(1.0-6.0)$ & $<0.001$ \\
\hline Hospital LOS, days (IQR) & $8(3.0-19.0)$ & $20(10.0-35.0)$ & $4(1.0-9.0)$ & $<0.001$ \\
\hline Serum Cr, mmol/L (IQR) & $1.4(1.0-2.2)$ & $1.3(1.0-1.6)$ & $1.5(1.1-2.4)$ & 0.004 \\
\hline No-flow interval, min (IQR) & $10(5.0-15.0)$ & $8(2.0-10.0)$ & $10(5.0-15.0)$ & 0.020 \\
\hline Low-flow interval, min (IQR) & $18(9.0-31.0)$ & $16(6.0-29.0)$ & $18(11.0-33.0)$ & 0.050 \\
\hline Shockable rhythm [n (\%)] & $49(25.8)$ & $27(40.3)$ & $22(17.9)$ & 0.001 \\
\hline Serum lactate, mmol/ $/(\mathrm{QRR})$ & $7.75(4.4-11.4)$ & $4.6(3-8)$ & $9.2(6.4-12.4)$ & $<0.001$ \\
\hline \multirow[t]{2}{*}{ OHCA score (IQR) } & 50.4 & 43.2 & 55.6 & $<0.001$ \\
\hline & $(41.2-59.4)$ & $(30.5-50.0)$ & $(46.4-62.7)$ & \\
\hline APACHE II score & $34(26.0-39.0)$ & $26(19.0-30.0)$ & $38(32.0-41.0)$ & $<0.001$ \\
\hline APACHE III score & $106(74.0-125.0)$ & $63(48.0-86.0)$ & $118(102.0-133.0)$ & $<0.001$ \\
\hline SAPS II score & $60(49.0-71.0)$ & $44(36.0-55.0)$ & $67(58.0-76.0)$ & $<0.001$ \\
\hline SAPS 3 score & 77 (63.0-87.0) & 59 (55.0-69.0) & 83 (75.0-89.0) & $<0.001$ \\
\hline
\end{tabular}

IQR=interquartile range, *Other: cardiomyopathy, pulmonary embolism, electrical injury, hypovolemia, metabolic disturbance, cardiac tamponade, ROSC=return of spontaneous circulation, VA ECMO=veno-arterial extracorporeal membrane oxygenator, ICU LOS=intensive care unit length of stay, $\mathrm{Cr}=$ creatinine, $\mathrm{mmol} / \mathrm{L}=$ millimoles per liter, $\mathrm{OHCA}=$ out-of-hospital cardiac arrest, $\mathrm{APACHE}=\mathrm{Acute} \mathrm{Physiology}$ and Chronic Health Evaluation, SAPS=Simplified Acute Physiology Score 


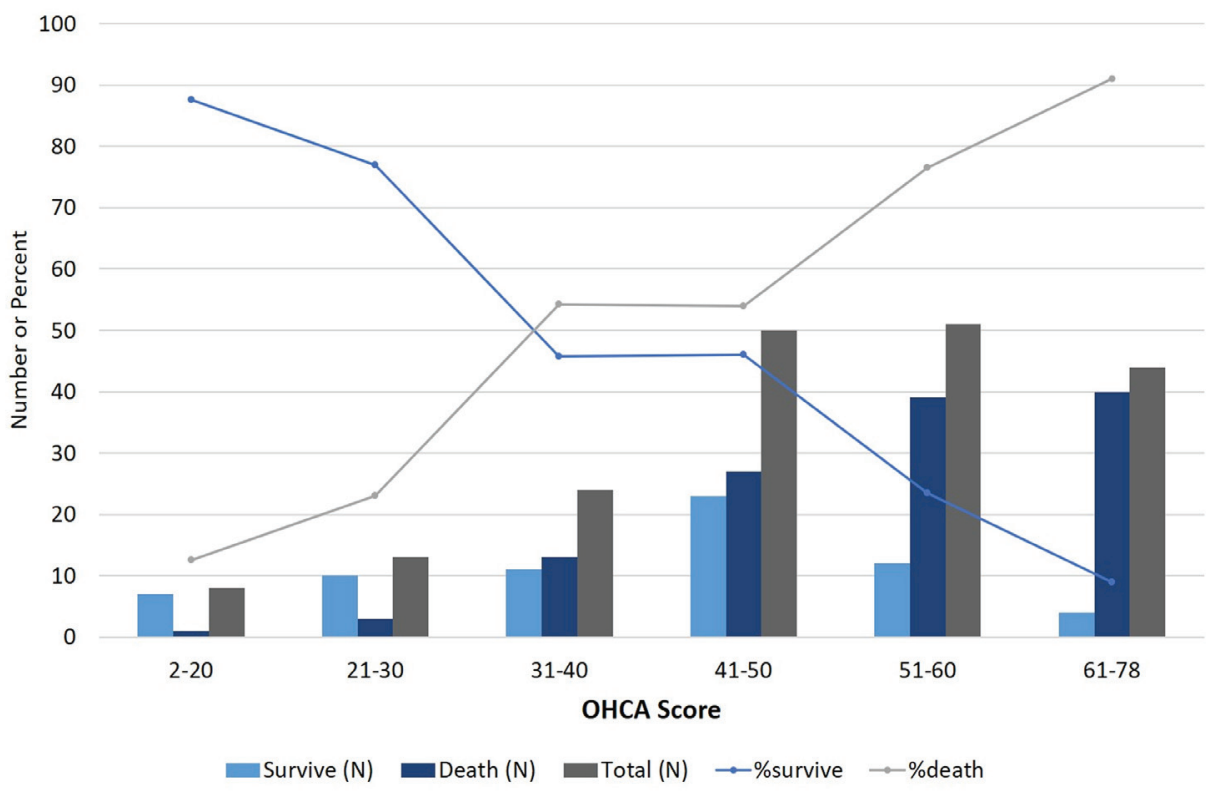

Figure 1 Hospital mortality rate arranged by the out-of-hospital cardiac arrest score

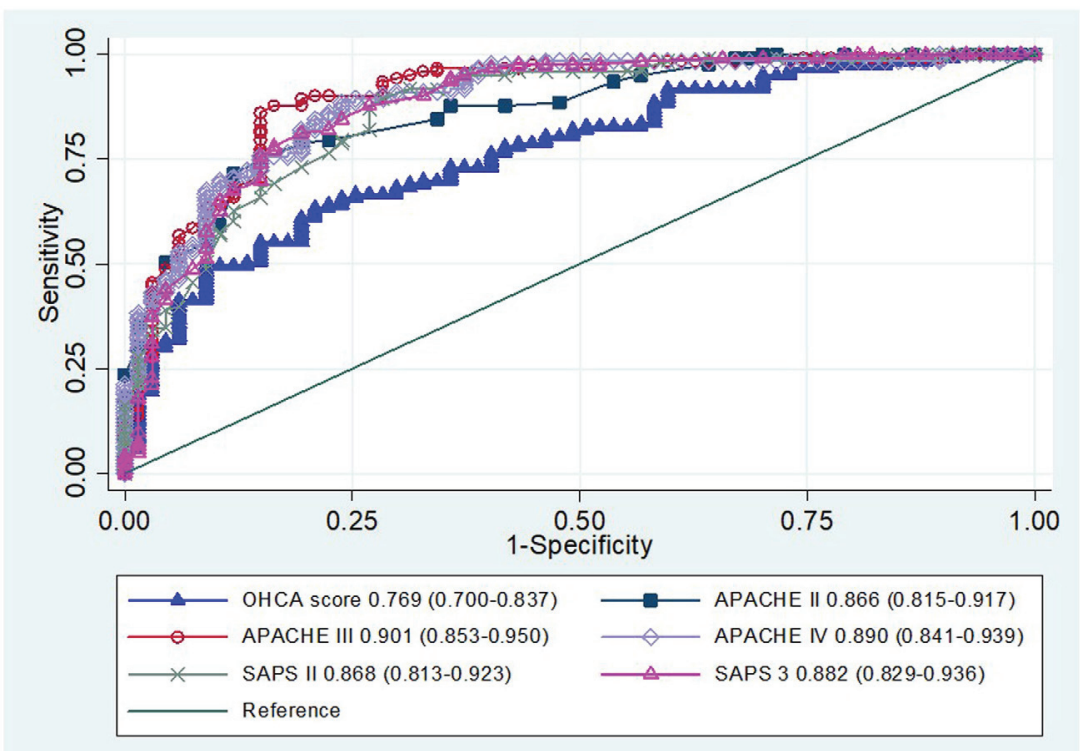

APACHE=Acute Physiology and Chronic Health Evaluation, SAPS=Simplified Acute Physiology Score, OHCA=out-of-hospital cardiac arrest

Figure 2 Comparison of the area under the receiver operating characteristic curves of the out-of-hospital cardiac arrest score with other severity scores for hospital mortality prediction in out-of-hospital cardiac arrest with return of spontaneous circulation patients admitted in intensive care unit. 
The actual hospital mortality rate was $64.7 \%$. The OHCA score predicted hospital mortality of $95.3 \pm 8.4$, which significantly overestimated the mortality with SMR 0.68 (95\% Cl 0.56-0.81). Meanwhile, the non-disease specific severity scores; APACHE II overestimated hospital mortality with SMR 0.83 (95\% Cl 0.69-0.99), in contrast, APACHE
IV underestimated hospital mortality with SMR 1.29 (1.071.54). The others (APACHE III, SAPS II and SAPS 3) presented good mortality prediction with $95 \% \mathrm{Cl}$ of SMR between 1. The calibration of the OHCA score and other non-disease specific severity scores were poor due to the $\mathrm{H}-\mathrm{L}$ goodness-of-fit test $<0.1$ (Table 2 and Figure 3).

Table 2 The performance of Out-of-Hospital Cardiac Arrest score, Acute Physiology and Chronic Health Evaluation II, III, IV, Simplified Acute Physiology Score II, and Simplified Acute Physiology Score

\begin{tabular}{llllll}
\hline & AUC $(\mathbf{9 5 \%} \mathbf{C l})$ & SMR $(\mathbf{9 5} \% \mathbf{C l})$ & H Chi-2, p-value & C Chi-2, p-value & Brier score \\
\hline OHCA score & $0.769(0.700-0.837)$ & $0.68(0.56-0.81)$ & $550.18,<0.001$ & $692.80,<0.001$ & 0.299 \\
APACHE II & $0.866(0.815-0.917)$ & $0.83(0.69-0.99)$ & $28.70,<0.001$ & $42.40,<0.001$ & 0.170 \\
APACHE III & $0.901(0.853-0.950)$ & $0.93(0.77-1.11)$ & $7.46,0.190$ & $19.95,0.001$ & 0.144 \\
APACHE IV & $0.890(0.841-0.939)$ & $1.29(1.07-1.54)$ & $29.87,<0.001$ & $29.85,<0.001$ & 0.153 \\
SAPS II & $0.868(0.813-0.923)$ & $1.04(0.87-1.24)$ & $4.95,0.420$ & $15.60,0.008$ & 0.136 \\
SAPS 3 & $0.882(0.829-0.936)$ & $1.08(0.90-1.29)$ & $6.93,0.220$ & $14.75,0.010$ & 0.127 \\
\hline
\end{tabular}

APACHE=Acute Physiology and Chronic Health Evaluation, AUC=area under the receiver operating characteristic, C=Hosmer-Lemeshow goodness-of-fit $\mathrm{C}$ test, $\mathrm{Cl}=$ confidence interval, $\mathrm{H}=$ Hosmer-Lemeshow goodness-of-fit $\mathrm{H}$ test, OHCA score=Out-of-Hospital Cardiac Arrest score, SAPS=Simplified Acute Physiology Score, SMR=standardized mortality ratio

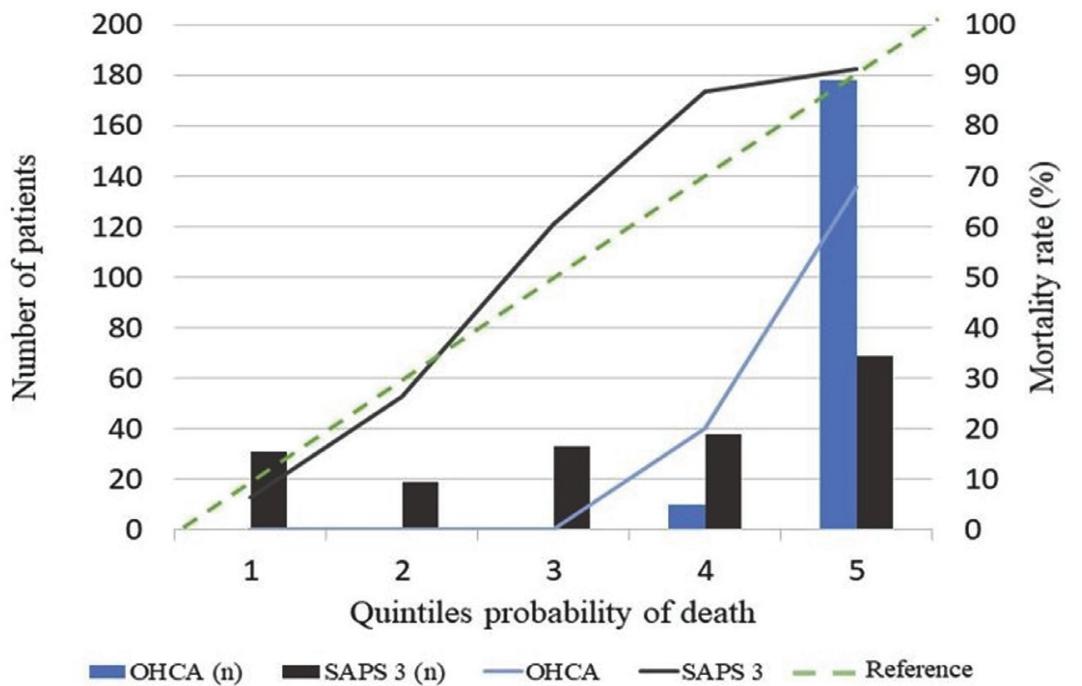

OHCA=out-of-hospital cardiac arrest, SAPS 3=Simplified Acute Physiology Score 3

Figure 3 Calibration curve for the out-of-hospital cardiac arrest score and Simplified Acute Physiology Score 3. 


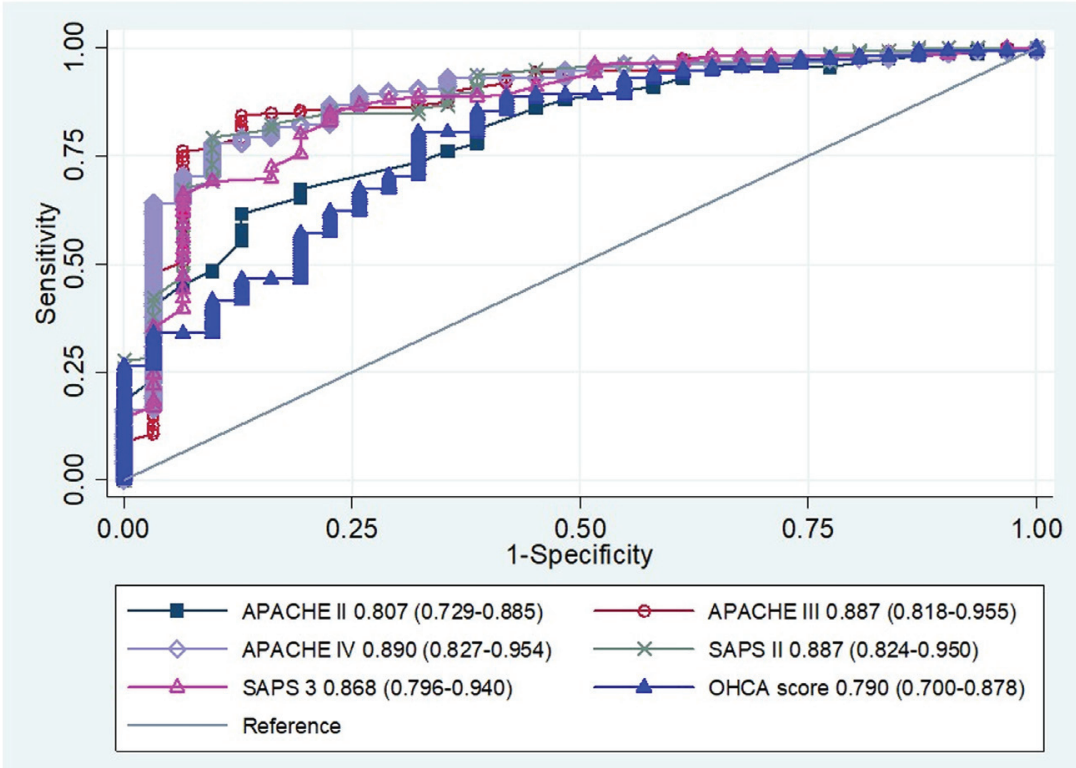

APACHE=Acute Physiology and Chronic Health Evaluation, SAPS=Simplified Acute Physiology Score, OHCA=out-of-hospital cardiac arrest

Figure 4 Comparison of the area under the receiver operating characteristic curves of the out-of-hospital cardiac arrest score with other severity scores for unfavorable neurological outcome in out-of-hospital cardiac arrest with return of spontaneous circulation patients admitted in intensive care unit.

Overall performance was evaluated by Brier scores, representing that OHCA score was objectionable, with the highest Brier score being 0.299. Meanwhile, SAPS 3 revealed the best overall performance with a Brier score of 0.127. The remaining ranking of overall performance was SAPS II, APACHE III, APACHE IV, APACHE II with Brier scores of $0.136,0.144,0.153,0.170$, respectively.

We obtained a secondary outcome to predict unfavorable neurological consequence categorized by $\mathrm{CPC}$, the OHCA score had moderate discrimination to predict poor neurological consequence with the AUC 0.790 (95\% Cl 0.700-0.878). Other non-disease specific severity scores had good discrimination with the AUC more than 0.8, APACHE IV had the best discrimination with the AUC 0.890 (95\% Cl 0.827-0.954) (Figure 4).

\section{Discussion}

Our study found the OHCA score had moderate discrimination for predicting hospital mortality in case of OHCA with ROSC admitted to ICU. The OHCA score was originated by Adrie et al., they reported AUCs of 0.82 in the development cohort and 0.88 in the validation cohort and showed well calibration by the goodness-of-fit test. ${ }^{17}$ Our results of the OHCA score were different compared to the original OHCA research that may be due to time difference in terms of no flow and low flow intervals. The original study revealed that the mean of no-flow interval was 3 and 8 minutes, and mean of low-flow interval was 7.5 and 15 minutes in good and poor outcome groups, respectively. In contrast, our study showed that the mean of no-flow interval was 8 and 10 minutes, and mean of 
low-flow interval was 16 and 18 minutes in survivors and non-survivors, consecutively. It was clearly seen that the population in our study experienced more prolonged resuscitation duration than the original study population. However, the hospital mortality in the present study was lower, which may be due to the improvement of knowledge and physicians' experience in each time period and the CPR guideline which was launched in a different time period. The CPR guideline is always updated every five years. In the original study, the data was collected from 1999 to 2003 while, the data in our study was collected from 2011 to 2019. Moreover, the population in this study may be less severe than in the original study because of lower severity score SAPS II.

Furthermore, in our study, APACHE II-IV, SAPS II and SAPS 3 had good discrimination. APACHE III had the best discrimination for predicting hospital mortality and SAPS 3 had the best overall performance. For secondary outcome, all non-disease specific severity scores had good discrimination. The reason why the OHCA score had lower discrimination for predicting mortality and poor neurological outcome than the other non-disease specific severity scores was the diversity in categorical variables. The OHCA score had only five variables with shockable rhythm, no flow time, low flow time, serum creatinine and lactate level. Meanwhile, the other non-disease specific severity scores had more variables comprise of several physiological parameters such as vital signs, severity of organ dysfunction, age, and comorbid diseases.

The AUC of the OHCA in the present study had moderate discrimination to predict hospital mortality, which was similar to two previous studies. In the studies by Skrifvars et al. $^{27}$ and Choi et al. ${ }^{28}$, the AUC of the OHCA score showed the AUC 0.77 and 0.74 , respectively. Skrifvars et al. investigated the survival and outcome using the APACHE III and the OHCA score in patients treated in the ICU following out-of-hospital, in-hospital or ICU cardiac arrest ${ }^{27}$. Choi et al. investigated the performance on the APACHE II, SAPS II, SOFA and the OHCA score of post-cardiac arrest patients treated with therapeutic hypothermia $^{28}$. However, the APACHE III in our study was better than the OHCA in discrimination, calibration and overall performance in contrast to the result by Skrifvars et al., in which the AUC of the OHCA was slightly better than the APACHE III. The reason may be caused by the different of study population, as Skrifvars et al. enrolled out-of-hospital, in-hospital and ICU cardiac arrest patients, whereas the present study included only OHCA patients following the original article developed for OHCA population. OHCA patients had several different factors compared to in-hospital or ICU cardiac arrest patients such as facilities, medical personal, and time detection to cardiac arrest. Furthermore, the APACHE II-IV, SAPS II and SAPS 3 in our study had good discrimination in both aspects of predicting mortality and unfavorable neurological consequence that were different to the previous study by Choi et al., which found that APACHE $\|$ and SAPS II revealed moderate discrimination for predicting mortality in post cardiac arrest patients treated with therapeutic hypothermia. ${ }^{28}$ The APACHE II and SAPS II were originated based on the worst values within the first 24 hours after ICU admission, but in the previous study by Choi et al. ${ }^{28}$, the APACHE II and SAPS II were calculated at the time of admission (0 hour), 24 and 48 hours from the admission time.

Our study suggested that SAPS 3 should be used for OHCA patients because of the best overall performance, good discrimination to predict hospital mortality and poor neurological outcome. In addition, this score was accessed simply by search engine and the variables were less sophisticated than APACHE III, despite the best discrimination to predict hospital mortality of APACHE III. In addition, the performance of the OHCA score may be 
ameliorated by secondary customization applying new variable parameters such as vital signs, age, preexisting comorbidities and new physiological variables.

The strength of the present study was, so far as we know, this is the first study that investigated all versions of broad commonly used severity scores such as APACHE II, III, IV, SAPS II and SAPS 3, for the general intensive care population compared to disease specific OHCA score. Secondly, the present study is the first attempt to examine not only discrimination but also calibration and overall performance of each severity score. Furthermore, this study endeavored to examine entire patients with and without target temperature management.

There were some limitations of this study. Firstly, this was a retrospective study and may have risk of bias to verify outcome. Secondly, the calibration examined by $\mathrm{H}-\mathrm{L}$ goodness-of-fit test, this test may be underpowered especially when fewer than six groups are formed. ${ }^{29}$ Thirdly, usual knowledge during a different time period may be effect ICU standard care, the latest CPR guideline was modified and launched in 2015, meanwhile our study enrolled the population from January 2011 to September 2019.

\section{Conclusion}

All non-disease specific severity scores indicated superior overall performance and demonstrated good discrimination for predicting hospital mortality and unfavorable neurological consequence better than the OHCA score. SAPS 3 indicated the best overall performance. However, all scores revealed poor calibration. Therefore, specific scores for OHCA patients should be modified for predicting mortality and neurological consequence in patients.

\section{Acknowledgement}

The authors are grateful to Ms. Rattina Jomsuriya, Faculty of Medicine, Prince of Songkla University for her help in data collection. The authors would like to acknowledge
Geoffrey Cox, International Affairs Department, Faculty of Medicine, Prince of Songkla University for editing the draft of this manuscript.

\section{Funding sources}

None declared.

\section{Conflict of interest}

There are no potential conflicts of interest to declare.

\section{References}

1. Berdowski J, Berg RA, Tijssen JG, Koster RW. Global incidences of out-of-hospital cardiac arrest and survival rates: Systematic review of 67 prospective studies. Resuscitation 2010;81: $1479-87$.

2. Larribau R, Deham H, Niquille M, Sarasin FP. Improvement of out-of-hospital cardiac arrest survival rate after implementation of the 2010 resuscitation guidelines. PLoS One 2018; 13:e0204169.

3. Lemiale V, Dumas F, Mongardon N, Giovanetti O, Charpentier J, Chiche JD, et al. Intensive care unit mortality after cardiac arrest: the relative contribution of shock and brain injury in a large cohort. Intensive Care Med 2013;39:1972-80.

4. Mongardon N, Dumas F, Ricome S, Grimaldi D, Hissem T, Pene F, et al. Postcardiac arrest syndrome: from immediate resuscitation to long-term outcome. Ann Intensive Care 2011;1:45.

5. Moulaert VR, Verbunt JA, van Heugten CM, Wade DT. Cognitive impairments in survivors of out-of-hospital cardiac arrest: a systematic review. Resuscitation 2009;80:297-305.

6. Sandroni C, D'Arrigo S, Nolan JP. Prognostication after cardiac arrest. Crit Care 2018;22:150.

7. Hawkes MA, Rabinstein AA. Neurological prognostication after cardiac arrest in the era of target temperature management. Curr Neurol Neurosci Rep 2019;19:10.

8. Vincent JL, Moreno R. Clinical review: scoring systems in the critically ill. Crit Care 2010;14:207.

9. Rapsang AG, Shyam DC. Scoring systems in the intensive care unit: A compendium. Indian J Crit Care Med 2014;18:220-8.

10. Keegan MT, Gajic O, Afessa B. Severity of illness scoring systems in the intensive care unit. Crit Care Med 2011;39: 163-9. 
11. Breslow MJ, Badawi O. Severity scoring in the critically ill: part 1--interpretation and accuracy of outcome prediction scoring systems. Chest 2012;141:245-52.

12. Breslow MJ, Badawi O. Severity scoring in the critically ill: part 2: maximizing value from outcome prediction scoring systems. Chest 2012;141:518-27.

13. Khwannimit B, Bhurayanontachai R. The performance of customised APACHE II and SAPS I| in predicting mortality of mixed critically ill patients in a Thai medical intensive care unit. Anaesth Intensive Care 2009;37:784-90.

14. Ridley S. Severity of illness scoring systems and performance appraisal. Anaesthesia 1998;53:1185-94.

15. Le Gall JR. The use of severity scores in the intensive care unit. Intensive Care Med 2005;31:1618-23.

16. Sathaporn N, Khwannimit B. Validation the performance of New York Sepsis Severity Score compared with Sepsis Severity Score in predicting hospital mortality among sepsis patients. J Crit Care 2019;53:155-61.

17. Adrie C, Cariou A, Mourvillier B, Laurent I, Dabbane H, Hantala $F$, et al. Predicting survival with good neurological recovery at hospital admission after successful resuscitation of out-ofhospital cardiac arrest: the OHCA score. Eur Heart J 2006;27: 2840-5.

18. Knaus WA, Draper EA, Wagner DP, Zimmerman JE. APACHE II: a severity of disease classification system. Crit Care Med 1985;13:818-29.

19. LeGall JR, Loirat P, Alperovitch A. APACHE II--a severity of disease classification system. Crit Care Med 1986;14:754-5.

20. Knaus WA, Wagner DP, Draper EA, Zimmerman JE, Bergner $M$, Bastos PG, et al. The APACHE III prognostic system. Risk prediction of hospital mortality for critically ill hospitalized adults. Chest 1991;100:1619-36.
21. Zimmerman JE, Kramer AA, McNair DS, Malila FM. Acute Physiology and Chronic Health Evaluation (APACHE) IV: hospital mortality assessment for today's critically ill patients. Crit Care Med 2006;34:1297-310.

22. Le Gall JR, Lemeshow S, Saulnier F. A new simplified acute physiology score (SAPS II) based on a European/North American multicenter study. JAMA 1993;270:2957-63.

23. Metnitz PG, Moreno RP, Almeida E, Jordan B, Bauer P, Campos RA, et al. SAPS 3--From evaluation of the patient to evaluation of the intensive care unit. Part 1: objectives, methods and cohort description. Intensive Care Med 2005; 31:1336-44.

24. Paul M, Legriel S. Neurological prognostication after cardiac arrest: how the "Best CPC" project would overcome selection biases. Crit Care 2019;23:246.

25. Sandroni C, Geocadin RG. Neurological prognostication after cardiac arrest. Curr Opin Crit Care 2015;21:209-14.

26. Fischer JE, Bachmann LM, Jaeschke R. A readers' guide to the interpretation of diagnostic test properties: clinical example of sepsis. Intensive Care Med 2003;29:1043-51.

27. Skrifvars MB, Varghese B, Parr MJ. Survival and outcome prediction using the Apache III and the out-of-hospital cardiac arrest (OHCA) score in patients treated in the intensive care unit (ICU) following out-of-hospital, in-hospital or ICU cardiac arrest. Resuscitation 2012;83:728-33.

28. Choi JY, Jang JH, Lim YS, Jang JY, Lee G, Yang HJ, et al. Performance on the APACHE II, SAPS II, SOFA and the OHCA score of post-cardiac arrest patients treated with therapeutic hypothermia. PLoS One 2018;13:e0196197.

29. Khwannimit B, Bhurayanontachai R. A comparison of the performance of Simplified Acute Physiology Score 3 with old standard severity scores and customized scores in a mixed medical-coronary care unit. Minerva Anestesiol 2011;77:305-12. 